\title{
Using Group Knowledge for Multitarget Terrain-Based State Estimation
}

\author{
Edward Sobiesk ${ }^{1}$, Maria Gini² , and John A. Marin ${ }^{3}$ \\ 1 Dept of Electrical Engineering and Computer Science, United States Military \\ Academy, West Point, NY 10996 edward.sobiesk@us.army.mil \\ 2 Dept of Computer Science and Engineering, University of Minnesota, 200 Union \\ St. S.E., Minneapolis, MN 55455-0159 gini@cs.umn.edu \\ 3 BBN Technologies, Columbia, MD 21046 jamarin@bbn.com
}

Summary. Multitarget terrain-based tracking is a cyclic process that combines sensor information with state estimation and data association techniques to maintain an estimate of the state of an environment in which ground-based vehicles are operating. When the ground-based vehicles are military vehicles moving across terrain, most of them will being moving in groups instead of autonomously. This work presents a methodology that has been demonstrated to improve the estimation aspect of the tracking process for this military domain. A clustering algorithm identifies groups within a vehicular data set. Group characteristics are extracted and then a new, innovative technique is utilized to integrate these into the individual vehicles' state estimation process. A series of experiments shows that the proposed methodology significantly improves the performance of three classic estimation algorithms for multitarget terrain-based tracking.

\section{Introduction}

Multitarget tracking is essential for any surveillance system utilizing sensors to interpret an environment. A multitarget terrain-based tracking system attempts to maintain an accurate description of an environment in which ground-based vehicles are operating. This cyclic process involves state estimation and data association techniques as well as the fusion of intermittent sensor reports with a priori information [6]. This information includes both characteristics of the environment, such as detailed terrain information, and characteristics of the entities operating in the environment, such as the capabilities and movement techniques of the vehicles involved.

In this work, we present a methodology for improving the estimation process for the multitarget terrain-based tracking domain. We introduce algorithms that identify groups of military vehicles from within a data set, extract some of their group characteristics, and then integrate these characteristics into the individual vehicle estimation process. We present the results of 126 


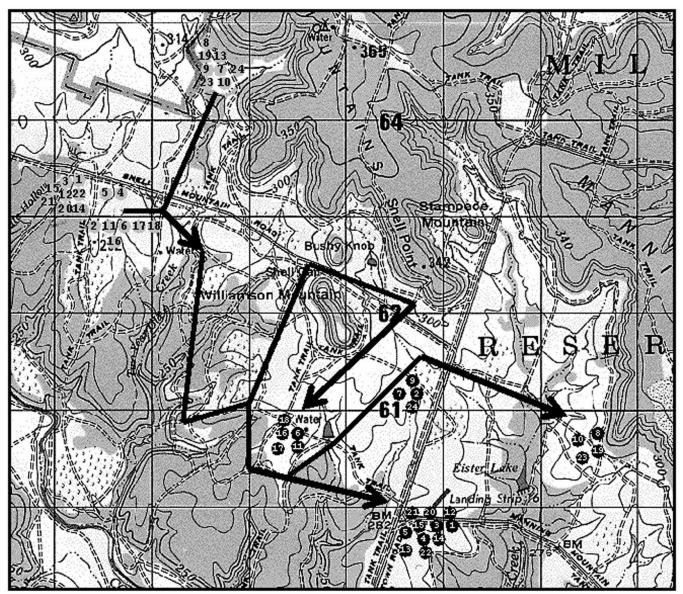

Fig. 1. Visualization of the movement routes taken by the 24 vehicles of Data Set One. The vehicles' start locations are marked by gray circles with black numbers, the finish locations by black circles with white numbers. The bold black arrows mark the general routes taken by the vehicles. Each grid square is 1000 meters long.

experiments conducted on large, real world data sets that test the methodology using three different state estimation models. In these experiments, an estimation model predicts the future locations of military vehicles as they move across terrain. The experiments compare the performance of the estimation models using group knowledge against their performance without it. Time intervals of 30,60, and 90 seconds between sensor reports are tested. Two different ways of computing group knowledge and three different methods of integrating group knowledge are examined. The estimation models used include an $\alpha-\beta$ Filter, a Kalman Filter, and an iterative State-Space Model. The three different data sets are recorded from actual maneuvers by U.S. Army tanks in a training area at Fort Hood, Texas. Fig. 1 visualizes the movements of one of the vehicular data sets.

The major contribution of this work is demonstrating experimentally that group knowledge improves individual vehicle state estimation. Critical to the use of group knowledge is an algorithm for identifying groups and an innovative method of integrating group knowledge into state estimation models. It is not our intent to identify the best estimation model for terrain-based tracking. By using diverse models with different data sets and varying time intervals, we show the validity and robustness of our methodology.

\section{Problem Background and Model Descriptions}

The prediction phase of multitarget terrain-based tracking is the principal component of this research. Historically, the most common method used for 
prediction has been the Kalman Filter family of algorithms. Numerous mathematical models besides the Kalman Filter exist including the $\alpha-\beta$, Bayes, and Least-Squares Filters. All of these are designed to predict the future location of an object based on past observations. These models are especially valuable for tracking aircraft or ships, where there is minimum change in velocity and direction. A major drawback to using these models for terrain-based tracking is that it is very difficult to integrate into the prediction process known terrain information for the areas involved. For instance, a basic Kalman Filter is incapable of integrating terrain constraints such as the observation that the entity is following a road or is headed toward an obstacle (a lake, a hill, etc.). A State-Space Model, which does include such terrain constraints, is therefore included as one of the models tested in this work.

For all the models, we assume the sensor reports for time $k$ contain the reported $x$ and $y$ coordinates in meters, the past direction of movement, and the velocity in meters per time interval. Both the past direction of movement and the velocity are calculated based on the location data at times $k-1$ and $k$. To ensure consistency during testing, we assume that all sensor reports correlate correctly with their next reported location.

For the $\alpha-\beta$ Filter implementation [2], a value of 1 is used for both the $\alpha$ and $\beta$ parameters because experimentation on a subset of the data found that the erratic movement and velocity of vehicles maneuvering across terrain made the long term target history of little value for near term estimation. The classic Kalman Filter was also implemented [3]. Based on previous work and for ease of implementation, both filters tracked a vehicle's $x$ and $y$ coordinate motion separately. The state of a vehicle at time $k$ is defined by the estimated location and estimated velocity.

The State-Space Model (S-S) we use is based on the Reid and Bryson State-Space Model [8] and includes enhancements by both Nougues [6] and the authors of this work. This model removes the Gaussian assumption used in the Kalman Filter and accounts for the effects of on-road and off-road conditions on a vehicle's movement as well as terrain effects on a vehicle's speed and direction of movement. The terrain is represented as an eight-way connected grid of equally sized cells, which allows movement from a given cell to any one of the eight adjacent neighboring cells. Each grid cell represents a $20 \times 20$ meter square area of terrain. The probability distribution for the sensor report is initially assigned across the appropriate cells. The model's prediction algorithm then manipulates the probability distribution based on terrain information associated with each grid cell, the vehicle's velocity, and the vehicle's past direction of movement. The terrain information used includes data on vegetation, slope, hydrology, roads, and obstacles acquired from the U.S. National Imagery and Mapping Agency. The calculation and integration of the terrain factors utilizes the method [6] and data [5] created by Nougues. 

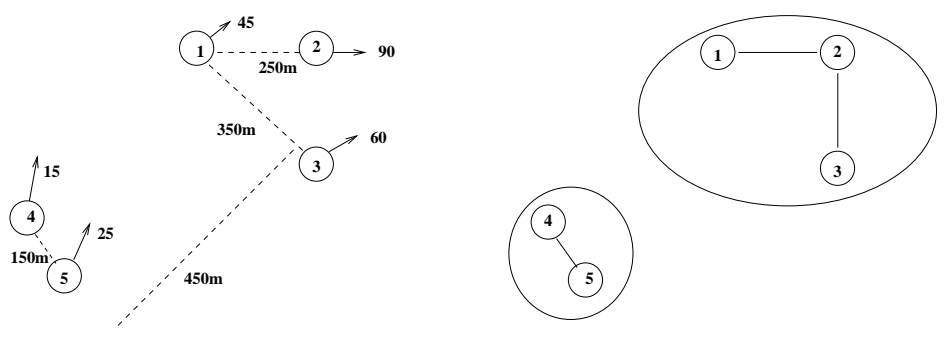

Fig. 2. Operation of the clustering algorithm. On the left is the relevant information about the vehicles derived from their sensor reports. On the right are the edges that result from applying the clustering criteria of $\delta=300$ meters and $\theta=50$ degrees to the vehicular data. Only three vehicular pairs meet the clustering criteria. Two groups of vehicles are formed by the connected components.

\section{Group Knowledge}

When multiple military vehicles move across terrain, most vehicles will be moving as part of a group instead of autonomously. This is evidenced by numerous U.S. Army manuals such as [1] that prescribe the different methods and formations to be used by groups of military vehicles. It is therefore logical to expect that information about the group would improve prediction.

For the sensor reports for time $k$, we define: A set of vehicles is a group if, and only if, each member vehicle is located within $\delta$ meters and has a past direction of movement within $\theta$ degrees of at least one other vehicle in the set. A simple example will illustrate this definition. Suppose vehicle 1 has a past direction of travel of 45 degrees, vehicle 2 of 90 degrees, and the vehicles are 200 meters apart. With $\delta=100$ and $\theta=50$, the vehicles are not a group. With $\delta=300$ and $\theta=25$, the vehicles are not a group. With $\delta=300$ and $\theta$ $=50$, the vehicles are a group.

Identification of Groups. Groups are identified by a clustering algorithm that does not require prior specification of the number of clusters and utilizes the distance between elements and the past direction of movement as the criteria for clustering. The algorithm we designed is inspired by the line clustering algorithm of Yin and Chen [12]. Our algorithm creates a graph with the vehicles as vertices. The graph is initialized to have no edges. All possible vehicle pairs are considered, and an edge is placed between every vehicle pair that meets the user specified distance and direction criteria. The final graph is formed once all possible vehicle pairs have been considered. The different connected components of the final graph are the desired groups of vehicles. Fig. 2 presents a simple example of the results of the algorithm.

Extraction of Group Knowledge. Once groups are identified, the following group characteristics are extracted: the central direction of movement, the overall velocity, the $x$ and $y$ coordinate velocities, the rank of every vehicle in the group based on their location relative to the projected central direction 


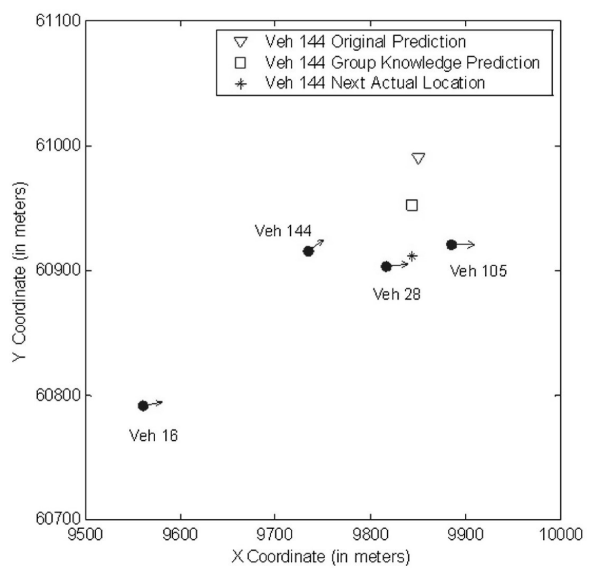

Fig. 3. Visualization of the application of $75 \%$ group knowledge adjusting the $\alpha$ - $\beta$ Filter's prediction for Vehicle 144 closer to its next actual location.

of movement, and the number of vehicles in the group. The group velocities and the central direction of movement are calculated either as the mean, or as the median, of the individual vehicles' characteristics. The rank of a vehicle in the group is its position within the group relative to the group's projected central direction of movement. A rank of 1 is assigned to the vehicle whose $x-y$ coordinate is furthest along the group's projected central direction of movement, a rank of 2 is assigned to the vehicle who is second furthest along, etc. The rank and the number of vehicles in the group are not integrated directly into any model, but are used together as part of a new method of determining the amount of group characteristic that is integrated into each vehicle's estimation.

Integration of Group Knowledge. We considered three methods for integrating group knowledge into the models:

1. the All-Group-Knowledge method uses only the calculated group characteristic and none of the individual vehicle characteristic;

2. the Half-Group-Knowledge method uses half of the group characteristic and half of the individual vehicle characteristic; and

3. the Varying-Group-Knowledge method uses different amounts of group characteristic for each vehicle in the group. This amount is calculated by dividing the vehicle's rank within the group by the number of vehicles in the group. This method assumes that lead vehicles in the central direction of movement are reflecting the future movement of the group more than the trail vehicles. Therefore, lead vehicles should use less of the group knowledge and more of their individual characteristic. Trail vehicles should use more of the group knowledge and less of their individual characteristic. Fig. 3 shows the results on Vehicle 144 whose rank is 3 out of 4 . 


\section{Experimental Results}

The three data sets used in the experiments are recorded from maneuvers by U.S. Army tanks at Fort Hood, Texas. The vehicular data consists of actual tank locations reported every 30 seconds. The locations were determined by mounting either a Global Positioning System or a Position Reporting and Recording System onto each vehicle. Both systems produce readings which are typically accurate to within a few meters. It is assumed that no goal destinations are known for the vehicles.

Data Set $\mathrm{One}^{4}$ consists of 24 vehicles moving during daylight across wooded trails and open terrain in a variety of formations with dynamically changing groups and group sizes. There are a total of 2007 individual moving sensor reports during the 72 minute movement. The average speed is 107.5 meters per 30 second time interval with a standard deviation of 64.1.

Data Set $\mathrm{Two}^{5}$ consists of 15 vehicles moving during darkness across wooded trails and open terrain almost exclusively in column and staggered column formations. Data Set Two remains a single group for most of the movement. There are a total of 1725 individual moving sensor reports during the 72 minute movement. The average speed is 78.5 meters per 30 second time interval with a standard deviation of 44.7 .

Data Set Three consists of 12 vehicles moving during daylight across wooded trails and open terrain. There are a variety of formations with moderate changes in group composition and size. There are a total of 405 individual moving sensor reports during the 21 minute movement. The average speed is 136.1 meters per 30 second time interval with a standard deviation of 71.9.

Throughout their movements, the vehicles in the above three data sets did not conduct any evasive maneuvers based on contact with an enemy.

An experiment consisted of employing the original model without group knowledge and then employing the same model 12 more times with the exact same data using all combinations of $\delta$ values of 100,300, and 500 meters and $\theta$ values of 25,50, 75, and 180 degrees. Experiments were conducted with time intervals of 30,60, and 90 seconds between sensor reports. Only moving sensor reports were tested.

The overall results are presented as the average Root Mean Square Error between a model's predicted location and the next reported location for all individual predictions made for the data set. To statistically compare the results of runs that used group knowledge against the ones that did not, paired one-tail t-tests were conducted using cutoff values of $5 \%$ and $1 \%$.

\footnotetext{
${ }^{4}$ Battalion Size Tank Movement Conducted at Ft. Hood, vehicular digital data files from U.S. Army Texcom, Fort Hood, TX, 1994.

${ }^{5}$ Initial Operational Test and Evaluation of the M1A2 Tank, vehicular digital data files from U.S. Army Texcom, Fort Hood, TX, 1993. This data source produced Data Sets Two and Three.
} 


\subsection{Selecting the Group Knowledge Integration Method}

81 experiments were conducted using the three state estimation models and the three data sets. Each experiment compared the use of no group knowledge against the use of the All-Group-Knowledge, Half-Group-Knowledge, or Varying-Group-Knowledge method. For all 81 experiments, group characteristics were calculated as the arithmetic mean. All-Group-Knowledge achieved at least a $5 \%$ statistical improvement over the original model without group knowledge $72.53 \%$ of the time, Half-Group-Knowledge $95.37 \%$, and VaryingGroup-Knowledge 96.30\%.

All three group knowledge integration methods provided substantial statistical improvement. The performance of the Half-Group-Knowledge and Varying-Group-Knowledge methods was particularly stunning as statistical improvement was achieved with over $95 \%$ of the different parameter values. To determine which integration method performs most robustly, the average percentage of improvement by the different methods was calculated for each model and for each time interval. Fig. 4 shows the results of this comparison for all 81 experiments.

\begin{tabular}{|c|c|c|c|c|c|c|c|c|}
\hline & \multicolumn{3}{|c|}{30 seconds } & \multicolumn{3}{|c|}{60 seconds } & \multicolumn{2}{|c|}{90 seconds } \\
\hline & $\alpha-\beta$ & Kalmar & S-S & $\alpha-\beta \mathrm{I}$ & Kalman & S-S & $\alpha-\beta$ & \begin{tabular}{|l|l} 
Kalman & S-S
\end{tabular} \\
\hline All-Group & $1.54 \%$ & $4.60 \%$ & $-0.15 \%$ & $7.64 \%$ & $4.88 \%$ & $1.45 \%$ & $9.25 \%$ & \begin{tabular}{|l|l|l}
$5.85 \%$ & 2.40 \\
\end{tabular} \\
\hline Half-Group & $6.48 \%$ & $5.39 \%$ & $1.29 \%$ & $7.47 \%$ & $4.43 \%$ & $1.95 \%$ & $7.48 \%$ & \begin{tabular}{l|l}
$4.88 \%$ & $2.21 \%$ \\
\end{tabular} \\
\hline Varying-Grou] & $6.62 \%$ & \begin{tabular}{|l|}
$9.24 \%$ \\
\end{tabular} & $1.23 \%$ & $9.24 \%$ & $7.08 \%$ & $2.44 \%$ & $9.78 \%$ & \begin{tabular}{|l|l|}
$8.24 \%$ & $2.79 \%$ \\
\end{tabular} \\
\hline
\end{tabular}

Fig. 4. Average percentages of improvement by the different integration methods over the original model employed without group knowledge.

Using the average percentage of improvement for comparison, VaryingGroup-Knowledge is the most robust of the methods considered. The VaryingGroup-Knowledge method performed better than the other two methods in eight out of the nine comparisons and was a very close second in the ninth. The Varying-Group-Knowledge method also demonstrated a consistency the other two methods lacked. The All-Group-Knowledge method had difficulty at the 30 second time interval, and the Half-Group-Knowledge method had difficulty at the 90 second time interval. Complete details on the models and full experimental and performance results are reported in [10].

\subsection{Group Knowledge improves performance}

Based on the above, we performed 27 additional experiments using the Varying-Group-Knowledge integration method and this time using the arithmetic median to calculate the group knowledge. The experiments included the different combinations of the data sets, models, time intervals, and $\delta$ and 


\begin{tabular}{|l|c|c|c|c|c|c|c|}
\hline & $\begin{array}{c}\alpha-\beta \\
\text { mean }\end{array}$ & $\begin{array}{c}\alpha-\beta \\
\text { median }\end{array}$ & $\begin{array}{c}\text { Kalman } \\
\text { mean }\end{array}$ & $\begin{array}{c}\text { Kalman } \\
\text { median }\end{array}$ & $\begin{array}{c}\text { S-S } \\
\text { mean }\end{array}$ & $\begin{array}{c}\text { S-S } \\
\text { median }\end{array}$ & Avg \\
\hline 30 sec, 1\% impr & $94.44 \%$ & $83.33 \%$ & $100.00 \%$ & $100.00 \%$ & $58.33 \%$ & $58.33 \%$ & $82.41 \%$ \\
30 sec, 5\% impr & $5.56 \%$ & $8.33 \%$ & $0.00 \%$ & $0.00 \%$ & $30.56 \%$ & $25.00 \%$ & $11.58 \%$ \\
30 sec, no impr & $0.00 \%$ & $8.34 \%$ & $0.00 \%$ & $0.00 \%$ & $11.11 \%$ & $16.67 \%$ & $6.02 \%$ \\
\hline 60 sec, 1\% impr & $97.22 \%$ & $97.22 \%$ & $100.00 \%$ & $97.22 \%$ & $91.67 \%$ & $91.67 \%$ & $95.83 \%$ \\
60 sec, 5\% impr & $0.00 \%$ & $0.00 \%$ & $0.00 \%$ & $2.78 \%$ & $5.56 \%$ & $0.00 \%$ & $1.39 \%$ \\
60 sec, no impr & $2.78 \%$ & $2.78 \%$ & $0.00 \%$ & $0.00 \%$ & $2.78 \%$ & $8.33 \%$ & $2.78 \%$ \\
\hline 90 sec, 1\% impr & $88.89 \%$ & $88.89 \%$ & $100.00 \%$ & $97.22 \%$ & $86.11 \%$ & $80.56 \%$ & $90.28 \%$ \\
90 sec, 5\% impr & $2.78 \%$ & $2.78 \%$ & $0.00 \%$ & $0.00 \%$ & $5.56 \%$ & $8.33 \%$ & $3.24 \%$ \\
90 sec, no impr & $8.33 \%$ & $8.33 \%$ & $0.00 \%$ & $2.78 \%$ & $8.33 \%$ & $11.11 \%$ & $6.48 \%$ \\
\hline Avg, 1\% impr & $93.52 \%$ & $89.81 \%$ & $100.00 \%$ & $98.15 \%$ & $78.70 \%$ & $76.85 \%$ & $89.51 \%$ \\
Avg, 5\% impr & $2.78 \%$ & $3.70 \%$ & $0.00 \%$ & $0.93 \%$ & $13.89 \%$ & $11.11 \%$ & $5.40 \%$ \\
Avg, no impr & $3.70 \%$ & $6.48 \%$ & $0.00 \%$ & $0.93 \%$ & $7.41 \%$ & $12.04 \%$ & $5.09 \%$ \\
\hline
\end{tabular}

Fig. 5. Percentages of experimental runs, from all experiments that used VaryingGroup-Knowledge, that achieved statistical improvement over the original model.

$\theta$ parameter values. In the 54 total experiments using the Varying-GroupKnowledge integration method, $89.51 \%$ achieved a statistical improvement over the original model at the $1 \%$ level and an additional $5.40 \%$ achieved a statistical improvement at the $5 \%$ level. The percentages of experimental runs that showed statistical improvement were calculated for each time interval and model. The results are in Fig. 5 .

The single most important observation of Fig. 5 is the consistent statistical improvement across all models and time intervals. This work used diverse models that assume different amounts of error. It used multiple data sets, time intervals, and methods of calculating the group characteristic. Despite all of these variables, group knowledge continually improved state estimation.

It is also essential to note that the above results are the average of all the $\delta$ - $\theta$ parameter values, but not all parameter values performed the same. For the $\alpha-\beta$ Filter and the State-Space Model, which treated the sensor reports as more accurate than the Kalman Filter did, two interesting trends occur. First, the best $\delta$ value distance becomes larger as the amount of time between sensor reports becomes longer. For 30 seconds, the best $\delta$ value is 100 meters, for 60 seconds it is 300 meters, and for 90 seconds it is 500 meters. The second interesting trend is that 180 degrees is generally the best $\theta$ value. This implies that information on vehicles moving in any direction provides improvement for these models. For the Kalman Filter, the best $\delta$ value is 500 meters for all three time intervals. The best $\theta$ values are generally always in the 25 or 50 degree range. These optimum parameter values for the Kalman Filter indicate that it is more sensitive to variation in past direction of movement than to proximity. 


\subsection{A case where Group Knowledge does not help}

An important aspect of any research is to establish the boundaries of applicability for the theory involved. This section presents the results of 18 experiments which demonstrate that in the special case when vehicles are conducting evasive maneuvers based on contact with an enemy, group knowledge does not generally improve the models' performance. Evasive maneuvers are actions taken by tanks when being fired upon by enemy vehicles. If evasive actions are conducted well, group knowledge will be of negligible assistance in prediction. To confirm this hypothesis, experiments were conducted using Data Set Four, which is a continuation of the movement of the 12 vehicles of Data Set Three as they conduct evasive maneuvers while in contact with an enemy. The 12 vehicles move during daylight across generally open terrain. There are a variety of formations with moderate changes in group composition and size. There are a total of 165 individual moving sensor reports during the 24 minute movement. The average speed is 107 meters per 30 second time period with a standard deviation of 75.8 .

18 experiments were conducted with Data Set Four, using the VaryingGroup-Knowledge method. Nine of the experiments calculated the group knowledge as the mean, and the other nine calculated it as the median. The experiments included the different combinations of the data sets, models, time intervals, and $\delta$ and $\theta$ parameter values. Only $19.4 \%$ of the experimental runs that used group knowledge showed statistical improvement over the original model. When in contact, tanks try to avoid being hit by enemy fire by taking such asymmetric actions as making radical turns, alternating speeds, and seeking cover and concealment in the terrain. These actions cause the characteristics of a group to be of little value in the estimation process.

\section{Related Work}

The iterative State-Space Model used in this work was designed by Reid and Bryson [8] and extended by Nougues [6], who demonstrated that it outperforms a Kalman Filter for terrain-based tracking of a single vehicle in a vegetative environment.

Pitman and Tenne [7] present a method of tracking ground-based vehicular convoys that combines convoy dynamics and topographical data to generate a probability density function that is used within a propagation algorithm. Their method also explores the influence a guide vehicle has on a trailing

vehicle. Sidenbladh and Wirkander [9] argue that due to the variability of terrain, multitarget terrain-based tracking is a non-linear problem for which Kalman approaches are inappropriate. They instead propose a particle filter for tracking a varying or unknown number of vehicles in terrain and visualize their method using a simulated scenario with three vehicles moving in terrain with human observation reports and five second time steps. Particle filters 
have become popular in many signal processing applications [4], as well as in robotics [11], since they do not require any assumption about the probability distributions of the data.

\section{Conclusions and Future Work}

We presented a methodology that improves existing multitarget terrain-based state estimation models by adding group knowledge. Using a vehicle's location within its group to determine the amount of group knowledge to integrate into the estimation process produced the best results. Experiments were conducted with large data sets recorded from actual vehicle movements. The results demonstrate that group knowledge significantly improves state estimation except when the vehicles are conducting evasive maneuvers based on contact with the enemy. Important areas of future research include testing the methodology with shorter time periods between sensor reports, using group knowledge to improve the data association aspect of tracking, exploring additional heuristics for determining the optimal amount of group knowledge to use, and adapting and testing this methodology with other models, such as a particle filter.

\section{References}

1. Field manual 17-15: Tank platoon, 1996. U.S. Army Armor Center, ATTN: ATSB-SBB-D, Fort Knox, KY 40121.

2. E. Brookner. Tracking and Kalman Filtering Made Easy. Wiley, 1998.

3. R. Brown and P. Hwang. Introduction to Random Signal Analysis and Kalman Filtering. John Wiley \& Sons, Inc., 1997.

4. A. Doucet, N. de Freitas, and N. Gordon, editors. Sequential Monte Carlo Methods in Practice. Springer Verlag, 2000.

5. P. Nougues. Terrain data files from Fort Hood, 1994. Terrain data files in raster format created at the Systems Engineering Dept, University of Virginia.

6. P. Nougues. Tracking Intelligent Objects in Terrain. PhD thesis, University of Virginia, 1996.

7. B. Pitman and D. Tenne. Tracking a convoy of ground vehicles. Technical report, State University of New York at Buffalo, January 2002.

8. D. Reid and R. Bryson. A non-Gaussian filter for tracking targets moving over terrain. In 12th Asilomar Conf. on Circuits, Systems, and Computers, 1978.

9. H. Sidenbladh and S. Wirkander. Tracking random sets of vehicles in terrain. In Proc. 2nd IEEE Workshop on Multi-Object Tracking, 2003.

10. Edward J. Sobiesk. Using Group Knowledge to track multiple vehicles moving across terrain. PhD thesis, University of Minnesota, 2000.

11. S. Thrun, D. Fox, W. Burgard, and F. Dellaert. Robust Monte Carlo localization for mobile robots. Artificial Intelligence, 128(1-2):99-141, 2001.

12. P. Yin and L. Chen. A new non-iterative approach for clustering. Pattern Recognition Letters, 15:125-133, 1994. 